\title{
A NOTE ON PSEUDOCOMPACT SPACES
}

\author{
JAMES E. JOSEPH
}

(Received 1 May 1978)

Communicated by J. H. Rubinstein

\begin{abstract}
In this note we give several new characterizations of arbitrary pseudocompact spaces, that is spaces characterized by the property that all continuous real-valued functions on the space are bounded.
\end{abstract}

Subject classification (Amer. Math. Soc. (MOS) 1970): primary 54 D 20, 54 C 30.

Keywords and Phrases: pseudocompactness, filterbases, complete accumulation points.

A topological space is pseudocompact if each continuous real-valued function on the space is bounded. Completely regular pseudocompact spaces have been studied extensively by Hewitt (1948), Glicksberg (1952, 1959), Bagley et al. (1958), Frolik (1960), Gillman and Jerison (1960), Scarborough and Stone (1966) and Comfort (1967) among others. By comparison, until recently, pseudocompact spaces which are not necessarily completely regular had been studied primarily by Stephenson (1968). The author (1978) has recently studied such spaces. In this note we offer several new characterizations, two of which utilize a generalization of the notion of complete accumulation point. We also employ a characterization of pseudocompactness introduced by the author (1978). We represent the class of continuous realvalued functions on a space $Y$ by $\mathscr{C}(Y)$. A point $y$ in a space $Y$ is in the closure of $a$ subset $K$ of the space with respect to $g \in \mathscr{C}(Y)(y \in \mathrm{cl}(K)(\bmod g))$ if $K \cap g^{-1}(H) \neq \varnothing$ for each $H$ open about $g(y) ; K \subset Y$ is closed with respect to $g \in \mathscr{C}(Y)($ closed $(\bmod g))$ if $\operatorname{cl}(K)(\bmod g) \subset K ; y$ is in the adherence of a filterbase $\Omega$ on $Y$ with respect to $g \in \mathscr{C}(Y)(y \in \operatorname{ad} \Omega(\bmod g))$ if $y \in \operatorname{cl}(F)(\bmod g)$ for each $F \in \Omega$. We will say that a filterbase $\Omega$ converges to $y$ with respect to $g \in \mathscr{C}(Y)(\Omega \rightarrow y(\bmod g))$ if for each $H$ open about $g(y)$ there is an $F \in \Omega$ with $F \subset g^{-1}(H)$. If $g \in \mathscr{C}(Y)$ and $K \subset Y$ then $c l(K)(\bmod g)$ is closed $(\bmod g)$. 


\section{The characterizations}

Pseudocompact spaces have been characterized by the author (1978) as those spaces $Y$ in which every filterbase (countable filterbase) $\Omega$ on the space satisfies $\operatorname{ad} \Omega(\bmod g) \neq \varnothing$ for each $g \in \mathscr{C}(Y)$.

Our first characterization parallels characterizations for minimal Hausdorff spaces by Herrington and Long (1975) and for $H$-closed spaces by the author (1977).

THEOREM 1. The following statements are equivalent for a space $Y$ :

(a) $Y$ is pseudocompact.

(b) For each $g \in \mathscr{C}(Y)$, each filterbase on $Y$ with at most one adherent point (modg) converges (modg).

(c) For each $g \in \mathscr{C}(Y)$, each countable filterbase on $Y$ with at most one adherent point (modg) converges $(\bmod g)$.

Proof that (a) ImPlies (b). Let $Y$ be pseudocompact, let $g \in \mathscr{C}(Y)$, and let $\Omega$ be a filterbase on $Y$ with at most one adherent point $(\bmod g)$, say $y$. If $\Omega \leftrightarrow y(\bmod g)$ there is a set $H$ open about $g(y)$ for which $\Omega^{*}=\left\{F \cap\left(Y-g^{-1}(H)\right): F \in \Omega\right\}$ is a filterbase on $Y$. So ad $\Omega^{*}(\bmod g) \neq \varnothing$ since $Y$ is pseudocompact. This implies that $y \in \operatorname{ad} \Omega(\bmod g) \cap c l\left(Y-g^{-1}(H)\right)(\bmod g)$. This is a contradiction since

$$
\operatorname{cl}\left(Y-g^{-1}(H)\right)(\bmod g) \subset Y-g^{-1}(H) \text {. }
$$

Proof that (b) IMPlies (c). Obvious.

ProOf THAT (c) IMPLies (a). This follows obviously from the above stated characterization of the author (1978).

The proof is complete.

A point $y$ in a space $Y$ is a complete accumulation point of $K \subset Y$ if for each open set $V$ about $x$ the sets $K$ and $V \cap K$ have the same cardinality $(|K|=|V \cap K|)$. It is known that a space is compact if and only if each infinite subset of the space has a c.a.p. (Kelley (1955), p. 163). If $g \in \mathscr{C}(Y)$ we say that $y \in Y$ is a complete accumulation point of $K \subset Y$ with respect to $g$ (c.a.p. $(\bmod g))$ if $|K|=\left|K \cap g^{-1}(H)\right|$ for each $H$ open about $g(y)$.

THEOREM 2. The following statements are equivalent for a space $Y$ :

(a) $Y$ is pseudocompact.

(b) Each infinite subset of $Y$ has a c.a.p. $(\bmod g)$ for each $g \in \mathscr{C}(Y)$.

(c) Each countably infinite subset of $Y$ has a c.a.p. (modg) for each $g \in \mathscr{C}(Y)$.

Proof that (a) ImPlies (b). Let $Y$ be pseudocompact, let $g \in \mathscr{C}(Y)$ and let $K \subset Y$ be infinite. Then $\Omega=\{K-H: H \subset K$ and $|H|<|K|\}$ is a filterbase on $Y$. Let 
$y \in a d \Omega(\bmod g)$ and let $H$ be open about $g(y)$. Then $g^{-1}(H) \cap\left(K-\left(K \cap g^{-1}(H)\right)\right)=\varnothing$. We conclude that $K-\left(K \cap g^{-1}(H)\right) \notin \Omega$ and, consequently, that $\left|K \cap g^{-1}(H)\right|=|K|$ So $y$ is a c.a.p. $(\bmod g)$ for $K$.

\section{Proof that (b) Implies (c). Obvious.}

Proof that (c) IMPLiES (a). Suppose that $Y$ is not pseudocompact. Then there is a countable filterbase $\Omega=\{F(j)\}_{j=1}^{\infty}$ and a $g \in \mathscr{C}(Y)$ with $\operatorname{ad} \Omega(\bmod g)=\varnothing$. We may assume without loss that $F(j) \subset F(k)$ when $j \geqslant k$. If $F(j)$ is finite for some $j$ we may choose for each $x \in F(j)$ a $j_{x}$ with the ultimate effect that

$$
\bigcap_{x \in F(j)} F\left(\mathrm{j}_{x}\right) \cap F(\mathrm{j})=\varnothing .
$$

This contradicts the fact that $\Omega$ is a filterbase. Hence $F(j)$ is infinite for each $j$. Inductively, choose $x(1) \in F(1)$ and for $j>1$ choose $x(\mathrm{j}) \in F(\mathrm{j})-\{x(k): k<j\}$. Let $K=\{x(\mathrm{j}): j=1,2,3, \ldots\}$ and let $x$ be a c.a.p. $(\bmod g)$ of $K$. Consider $F(k) \in \Omega$ and let $H$ be open about $g(x)$. There is a $j>k$ with $x(\mathrm{j}) \in g^{-1}(H)$, since $\left|K \cap g^{-1}(H)\right|=|K|$. So $g^{-1}(H) \cap F(k) \neq \varnothing$. Hence $x \in a d \Omega(\bmod g)$, a contradiction.

The proof is complete.

\section{References}

R. W. Bagley, E. H. Connell and J. D. McKnight, Jr. (1958), 'On properties characterizing pseudocompact spaces', Proc. Amer. Math. Soc. 9, 500-506.

W. W. Comfort (1967), 'A nonpseudocompact product space whose finite subproducts are pseudocompact', Math. Ann. 170, 41-44.

Z. Frolik (1960), 'The topological products of two pseudo compact spaces', Czechoslovak Math. J. 10, 339-349.

L. Gillman and M. Jerison (1960), Rings of Continuous Functions (Van Nostrand, New York).

I. Glicksberg (1952), 'The representation of functionals by integrals', Duke Math J. 19, 253-261.

I. Glicksberg (1959), 'Stone Cech compactifications of products', Trans. Amer. Math. Soc. 90, 369-382.

L. L. Herrington and P. E. Long (1975), 'Characterizations of C-compact spaces', Proc. Amer. Math. Soc. 52, 417-426.

E. Hewitt (1948), 'Rings of real-valued continuous functions', Trans. Amer. Math. Soc. 64, 45-99.

J. E. Joseph (1977), 'More characterizations of H-closed spaces', Proc. Amer. Math. Soc. 63, $160-164$.

J. E. Joseph (1978), 'Pseudocompactness and closed subsets of products', Proc. Amer. Math. Soc. (to appear).

J. L. Kelley (1955), General Topology (Van Nostrand, Princeton).

R. M. Stephenson, Jr. (1968), 'Pseudocompact spaces', Trans. Amer. Math. Soc. 134, 437-448.

\section{Department of Mathematics}

Howard University

Washington, D.C. 20059

U.S.A. 